\title{
ARTICLE
}

\section{Dopamine in the oval bed nucleus of the stria terminalis contributes to compulsive responding for sucrose in rats}

\author{
Amanda C. Maracle ${ }^{1}$, Catherine P. Normandeau ${ }^{2,3}$, Éric C. Dumont ${ }^{2,3}$ and Mary C. Olmstead ${ }^{1,2,3}$
}

\begin{abstract}
Binge eating disorder (BED) is characterized by periods of excessive food intake combined with subjective feelings of loss of control. We examined whether sucrose bingeing itself leads to uncontrolled or compulsive responding and whether this effect is magnified following a period of abstinence. We then assessed dopamine (DA) modulation of inhibitory synaptic transmission in the oval bed nucleus of the stria terminalis (ovBNST) as a neural correlate of compulsive responding and whether this behavioral effect could be disrupted by DA blockade in the ovBNST. Over 28 days, male Long-Evans rats ( $n=8-16$ per group) had access to $10 \%$ sucrose and food ( 12 or $24 \mathrm{~h}$ ), $0.1 \%$ saccharin and food $(12 \mathrm{~h})$, or food alone $(12 \mathrm{~h})$. Compulsive responding was assessed following 1 or 28 days of sucrose abstinence using a conditioned suppression paradigm. Only rats given $12 \mathrm{~h}$ access to sucrose developed binge-like intake, manifested as copious intake within the first hour; compulsive responding was significantly elevated in this group following 28 days of abstinence. In parallel, the effect of DA on ovBNST inhibitory transmission switched from a reduction to a potentiation; the effect, although observable after 1 day, was more pronounced and sustained following 28 days of abstinence. Intra-ovBNST infusions of a DA D1 receptor antagonist $(0.8 \mu \mathrm{g} / \mu \mathrm{l} \mathrm{SCH}-23390)$ reversed the blockade of conditioned suppression, thereby confirming the causal relationship between ovBNST DA modulation of $\gamma$-aminobutyric acid transmission and alterations in conditioned suppression following binge-like intake of sucrose.
\end{abstract}

Neuropsychopharmacology (2019) 44:381-389; https://doi.org/10.1038/s41386-018-0149-y

\section{INTRODUCTION}

Binge eating disorder (BED), the most common of all eating disorders [1, 2], is characterized by consuming larger amounts of food within a discrete period of time than would be expected under similar conditions [3]. Approximately 3\% of the general population are diagnosed with BED at any given time, although close to twice this number of American adults have a history of bingeing episodes [1]. Although binge eating may contribute to the growing obesity epidemic [4], only one-third of individuals who binge eat are obese or overweight. In contrast, more than three-quarters of BED patients are comorbid for other psychiatric conditions and close to $80 \%$ exhibit significant reductions in quality of life measures [5]. In addition, binge eating is a much higher predictor of addictive traits than is obesity or other eating-related dysfunctions [6-9]. Thus, regardless of whether binge eating meets the behavioral criteria of substance use disorders [10, 11], there is a pressing need to understand the etiology and mechanisms that drive this maladaptive behavior [12].

Animal models provide an opportunity to understand the etiology of binge eating in that behavioral characteristics of this condition are reproducible in rodents. For example, binge eating emerges following repeated, intermittent access to sucrose or high-fat food, as well as a history of dieting combined with stress [13]. An important characteristic of BED is loss of control over food intake: this compulsive eating continues despite knowledge of negative outcomes associated with bingeing [3]. Compulsion may be measured in animal models, therefore, as continued responding despite adverse consequences [14]. Extended access to a highfat diet produces this behavior in rats [15], although it is not clear whether sugar consumption produces the same effect. Thus, the first goal of our study was to examine whether sucrose bingeing leads to compulsive responding. We used a validated model of bingeing [16] in which rats have access to sucrose and chow for either 12 or $24 \mathrm{~h}$ per day. Within days, rats with restricted access (i.e., $12 \mathrm{~h}$ ) begin to consume copious amounts of sucrose in the first $1-2 \mathrm{~h}$ of exposure, mimicking binge-like eating patterns in humans. We then used the conditioned suppression (CS) paradigm to assess compulsive responding in these animals: we predicted that only rats with a history of sucrose bingeing would lever press for sucrose in the presence of a cue predicting an electrical foot shock.

We also tested the hypothesis that compulsive responding for sucrose would increase following a period of forced abstinence. Seeking responses for a cue paired with sucrose $[17,18]$, saccharin [19], cocaine [20], or heroin [21] increase in a time-dependent manner when the reinforcer is no longer available, a behavior that is reflected in underlying neurobiological changes (for a review, see ref. [22]). These studies provide evidence for incubation of drug or food craving; our study is the first to assess an incubation effect in the context of compulsive responding. We used a 28-day interval to match maximal sucrose-seeking responses following abstinence [17]. Understanding the incubation of compulsive responding has implications for developing strategies to deal with

\footnotetext{
${ }^{1}$ Department of Psychology, Queen's University, Kingston, ON K7L 3N6, Canada; ${ }^{2}$ Center for Neuroscience Studies, Queen's University, Kingston, ON K7L 3N6, Canada and ${ }^{3}$ Department of Biomedical and Molecular Sciences, Queen's University, Kingston, ON K7L 3N6, Canada Correspondence: Mary C. Olmstead (olmstead@queensu.ca)
}

Received: 4 April 2018 Revised: 6 June 2018 Accepted: 4 July 2018 Published online: 10 July 2018 
binge eating, as this behavior usually occurs following periods of food restriction (e.g., dieting) in humans.

In searching for a neurophysiological trace associated with compulsive sucrose intake, we focused on the oval subregion of the bed nucleus of the stria terminalis (ovBNST), which may contribute to feeding behaviors and binge eating. Indeed, pharmacological and optogenetic manipulations of the ovBNST robustly modulate feeding in rodents and stress-induced binge eating is associated with neurophysiological deregulation in the ovBNST [23-27]. Moreover, corticotropin-releasing hormone in the ovBNST is normally anorectic, but promotes binge eating following frustration-induced stress [27]. Likewise, dopamine (DA) has opposite effects on inhibitory synaptic transmission in the ovBNST of rats that do and do not have a history of cocaine intake; we hypothesized that such a change might also represent a neurophysiological trace of compulsive sucrose intake [28-31]. Finally, we explored a causal relationship between DA activity in the ovBNST and compulsive responding for sucrose by testing whether microinfusions of a selective DA D1 receptor (DRD1) antagonist (SCH-23390) into this region would disrupt compulsive responding that develops following binge sucrose intake.

\section{MATERIALS AND METHODS}

\section{Animals}

Two hundred and twenty-six male Long-Evans rats (Charles River Laboratories) were paired housed in standard polycarbonate cages on a 12/12 h light cycle (lights off at 0400 hours), until 1 day before the sucrose consumption sessions. At that point, all animals were singly housed in order to obtain precise measures of intake for individual subjects. Animals had ad libitum access to water in their home cage; food access (standard chow) was restricted to $2 \mathrm{~h}$ per day during operant sessions (so that animals were motivated to perform the task), and to 12 or $24 \mathrm{~h}$ per day during sucrose consumption sessions. All procedures were conducted in accordance with the Canadian Council of Animal Care guidelines and all experiments were approved by the Queen's University Animal Care Committee.

\section{Apparatus}

Operant training and testing were conducted in standard operant chambers (Med Associates, St. Albans, VT, USA). Each chamber was equipped with retractable levers, a reward delivery magazine (fitted with liquid dipper attachment), signal lights, a house light, shock capable flooring, and adjustable tone-producing speakers.

Weight and food consumption (g) were recorded with a standard scale. Liquid solutions were presented to rats in $100 \mathrm{ml}$ graduated glass drinking bottles fitted with rubber stoppers containing ball-tipped sipper tubes (Ancare Inc., Montréal, QC, Canada). Solution concentrations were $10 \%$ sucrose and $0.1 \%$ saccharin ( $\geq 99 \%$ purity, Sigma-Aldrich, Oakville, ON, Canada) in filtered water $(100 \mathrm{~g} / 1 \mathrm{~L}$ and $1 \mathrm{~g} / 1 \mathrm{~L}$, respectively).

\section{Procedures}

Three separate experiments were conducted, described below as behavior, electrophysiology, and microinfusion. All animals underwent sucrose consumption sessions and were then tested in behavior, electrophysiology, or microinfusion studies following either 1 or 28 days of sucrose abstinence. In the first two experiments (behavior and electrophysiology), separate groups of rats were tested at both time points; in the microinfusion study, all rats were tested after 1 day of abstinence. In the behavioral experiments, all group sizes were $n=8$ with the following exceptions: 1 -day abstinence, $12 \mathrm{~h}$ sucrose, $12 \mathrm{~h}$ food $n=16$ each; and 28-day abstinence, $24 \mathrm{~h}$ sucrose $n=9$. Electrophysiology group numbers (cells in brackets) following 1-day abstinence were as follows: $12 \mathrm{~h}$ sucrose $n=4(13), 12 \mathrm{~h}$ food $n=3(9) ; 12 \mathrm{~h}$ saccharin $n=3(7)$; and $24 \mathrm{~h}$ sucrose $n=2(6)$. Following 28 days of abstinence the values were as follows: $12 \mathrm{~h}$ sucrose $n=2(8) ; 12$ $\mathrm{h}$ food $n=2(7) ; 12 \mathrm{~h}$ saccharin $n=3(7)$; and $24 \mathrm{~h}$ sucrose $n=5$ (15). In the microinfusion experiment, all groups were $n=8$.

\section{Sucrose consumption}

The protocol for sucrose consumption, including solution concentrations and access periods, was based on a procedure that induces sucrose bingeing [16]. Briefly, animals were randomly assigned to one of four groups: intermittent sucrose $(12 \mathrm{~h}$ access to sucrose and food), intermittent saccharin (12 $\mathrm{h}$ access to saccharin and food), intermittent food ( $12 \mathrm{~h}$ access to food), or ad libitum sucrose ( $24 \mathrm{~h}$ access to sucrose and food). Each day for 28 days, beginning $4 \mathrm{~h}$ into the active cycle, animals were weighed and presented with solution and food. For the intermittent access groups, solution intake $(\mathrm{ml})$ was measured $1 \mathrm{~h}$ after presentation and on removal ( $12 \mathrm{~h}$ post presentation). For animals in the ad libitum group ( $24 \mathrm{~h}$ sucrose), solution intake was measured 1, 12, and $24 \mathrm{~h}$ after presentation. Food intake $(\mathrm{g})$ was measured at the end of the access period (12 or $24 \mathrm{~h}$ ).

\section{Abstinence}

Following sucrose consumption testing, equal numbers of animals from each group underwent either 1 or 28 days of abstinence in which they had no access to sucrose or saccharin solutions. During this period, animals were weighed regularly and restricted to $2 \mathrm{~h}$ of food access per day, provided $2-4 \mathrm{~h}$ after the beginning of the dark cycle. On this feeding schedule, body weights were maintained at $\sim 85 \%$, without failing below $80 \%$, of free-fed agematched controls.

\section{Experiment 1: Behavior}

Operant training. Subjects were trained to lever press for sucrose prior to the sucrose consumption sessions. The assignment of reinforced lever (right or left) was counterbalanced within groups. During training, both levers extended into the operant chamber and a house light (located at the back of the operant chamber) and cue light (above the reinforced lever) were illuminated. Each lever press on the reinforced lever elevated the liquid dipper (providing animals with access to $10 \%$ sucrose solution), turned off the house and cue lights, and turned on a "reward" light (located near the reward delivery magazine) for $5 \mathrm{~s}$. Over a 60-min session, animals could receive a maximum of 80 dipper elevations. Training continued until animals obtained $\geq 60$ dipper elevations (75\% success rate) on each of two consecutive training days. Animals were then moved to a fixed ratio-3 (FR-3) and then an FR5 schedule of reinforcement using the same criterion. The final session of FR- 5 training served as a baseline. Following abstinence (1 or 28 days), animals were given one 60 -min session to reestablish lever pressing on an FR-5 schedule (operant retraining sessions).

Conditioned suppression testing. Two days prior to operant retraining sessions, animals were habituated to a new environment (unfamiliar operant box with different lighting and contextual cues for $30 \mathrm{~min}$, twice per day) where they would experience CS-US pairings. Following operant retraining sessions, animals underwent CS training using a procedure described previously [14]. Briefly, after a 5-min lead-in period, animals experienced two 10-min presentations of an $85 \mathrm{~dB} 2900 \mathrm{~Hz}$ tone (CS), paired with 10 random $1 \mathrm{~s} 0.5 \mathrm{~mA}$ scrambled foot shocks (inter-trial interval $5 \mathrm{~min}$ ).

The following day (i.e., the day after operant retraining), animals were tested for CS of lever pressing in the operant boxes in which they were originally trained. After 5-min in the operant chamber, the lever was inserted and rats experienced six CS-on/CS-off presentations ( $2 \mathrm{~min}$ CS-on followed by $2 \mathrm{~min}$ CS-off). The lever was retracted during the last $1 \mathrm{~min}$ of the session for a total session time of $30 \mathrm{~min}$. Lever presses that met reward criteria 
(FR-5) resulted in the presentation of cue lights associated with sucrose during training sessions, but no sucrose delivery.

A separate control group ( $12 \mathrm{~h}$ sucrose CS Unpaired) underwent the same experimental procedure as the $12 \mathrm{~h}$ sucrose group, but did not learn the tone-shock association during CS training. That is, these animals were exposed to the same number of foot shocks (20 in total), randomly presented over an 80-min session (alternating 10-min tone-on and 10-min tone-off periods). Session length was increased to ensure that the density of foot shocks was low enough to prevent animals from developing fear conditioning to the tone-on phase, the tone-off phase, or the environment.

\section{Experiment 2: Electrophysiology}

Animals in the electrophysiology experiments underwent sucrose consumption and abstinence sessions prior to brain slice neurophysiology, but no operant training. Neurophysiology experiments were conducted $36 \mathrm{~h}$ after the last presentation of sucrose, saccharin, or food (1-day abstinence) or on the 29th day of abstinence (28-day abstinence). In other words, electrophysiological testing occurred on the same day that animals in the CS experiment were re-exposed to sucrose following abstinence.

Slice preparation and recording. Slice preparation and recording followed the protocol published by Krawczyk et al. [30]. Briefly, neurons were voltage clamped at $-70 \mathrm{mV}$ and $\mathrm{GABA}_{\mathrm{A}}$ inhibitory post-synaptic currents $\left(G_{A B A}\right.$-IPSCs) were pharmacologically isolated with the AMPA receptor antagonist DNQX $(50 \mu \mathrm{M})$. $G_{A B A_{A}}-I P S C$ were evoked by paired electrical stimulation $(0.1 \mathrm{~ms}$ duration, $50 \mathrm{~ms}$ interval) applied at $0.1 \mathrm{~Hz}$ using local fiber stimulation with tungsten bipolar electrodes placed in the ovBNST approximately $100-500 \mu \mathrm{m}$ dorsal of the recorded neuron. Stimulation intensity $(0.01-0.5 \mathrm{~mA})$ was adjusted to obtain approximately $50 \%$ of evoked GABA $A_{A}$ IPSCs maximum amplitude. After a minimum of 5 min stable baseline, DA $(1 \mu \mathrm{M})$ was bathapplied for $5 \mathrm{~min}$ and washed for at least $15 \mathrm{~min}$. DA-induced changes in peak amplitude (in percent) and paired-pulse ratio $\left(\mathrm{PPR}_{50 \mathrm{~ms}}\right)$ of IPSCs were recorded and measured. Visual guidance was provided by an upright transmitted light microscope (BX51W1, Olympus Canada, Richmond Hill, ON, Canada). Recordings were done using a Multiclamp 700B amplifier and a Digidata 1440A digitizer (Molecular Devices Scientific, Sunnyvale, CA, USA). Data were acquired and analyzed with Axograph $X$ running on Apple computers.

\section{Experiment 3: Microinfusion}

Animals in the microinfusion experiment were allowed to habituate to the facility for a minimum of 3 days prior to surgery. Following recovery, animals underwent operant training, sucrose consumption, and CS testing, using the protocols described previously. The DRD1 antagonist, SCH-23390 (Sigma-Aldrich, Milwaukee, WI, USA), was infused prior to conditioned suppressed testing, as outlined below.

Intracranial surgery. Subjects were anesthetized using a combination of isoflurane and oxygen (up to $5 \%$ isoflurane). Meloxicam ( $2 \mathrm{mg} / \mathrm{kg}$ subcutaneously) was administered prior to surgery; bupivacaine $(2 \mathrm{mg} / \mathrm{kg})$ was injected around the incision area. Bilateral cannulae guides (Plastic One Inc., Roanoke, VA, USA) were implanted $1 \mathrm{~mm}$ above the ovBNST $(-0.26 \mathrm{AP}, \pm 1.9 \mathrm{ML},-6.5 \mathrm{DV}$ coordinates relative to bregma) [32]. Acrylic dental cement and four jeweller's screws $(0.08 \times 0.125 \mathrm{in}$.) secured the guide cannulae in place. Subjects recovered under a heat lamp for a minimum of $1 \mathrm{~h}$ until they regained consciousness. They were moved to a recovery room and treated with Meloxicam $(2 \mathrm{mg} / \mathrm{kg})$ and antibiotic ointment on the incision area, once daily for a minimum of 3 days. They were moved back to the colony room when they had regained or surpassed their pre-surgical weight.
Intracranial infusions. Prior to CS testing, animals in each access group were randomly assigned to drug or saline conditions. Based on this, animals received bilateral infusions of a selective D1 antagonist ( $\mathrm{SCH}-23390$ dissolved in $0.9 \%$ physiological saline) at concentrations of either 0 (saline) or $0.8 \mu \mathrm{g} / \mu \mathrm{l} 10 \mathrm{~min}$ prior to testing, based on previous parameters [30]. Infusions were administered in volumes of $0.5 \mu \mathrm{l}$ per side at a maximum rate of $1 \mu \mathrm{l} / \mathrm{min}$, using an internal cannula connected to a Hamilton microsyringe infusion pump. Cannulae were left in place for $1 \mathrm{~min}$ after the infusion to allow drug diffusion.

Perfusion and histology. Following CS testing, animals were anesthetized (oxygen and isoflurane), and then euthanized through full body perfusion using $4 \%$ paraformaldehyde (PFA). Brains were extracted and kept in 4\% PFA solution for a minimum of $48 \mathrm{~h}$. Coronal brain sections $(40 \mu \mathrm{m})$ were sliced using a Cryostat (Leica CM1850) and stained using cresyl violet acetate to verify anatomical placement of the guide cannulae.

Data from 20 animals were removed from the microinfusion study for the following reasons: five were ill during the experiment, two were tested in boxes with technical breakdowns during the test, and 12 had incorrect cannulae placements. The remaining 64 animals were split evenly across sucrose access ( $12 \mathrm{~h}$ sucrose, $12 \mathrm{~h}$ food, $12 \mathrm{~h}$ saccharin, or $24 \mathrm{~h}$ sucrose) and microinfusion (saline or SCH-23390) groups ( $n=8$ each).

\section{Data analysis}

Sucrose consumption. All data are represented as a percent of body weight $(\mathrm{g} / \mathrm{g}$ or $\mathrm{ml} / \mathrm{g}$ based on the animals recorded weight each day). Data were analyzed using repeated-measures analysis of variance (RM ANOVA) with time as the repeated measure and group as the between-subjects measure. In cases in which sphericity was violated (Mauchley's $p<0.001$ ), the Greenhouse-Geisser correction was used (df rounded to the nearest whole number). Significant interactions were followed up with simple main-effects analyses and multiple pairwise comparisons using a Bonferroni correction. To analyze group differences, simple main-effects analyses were conducted comparing data on the first and last four days of sucrose consumption sessions.

Operant training. Lever pressing responses during baseline and retraining sessions were non-normal, so a bootstrapping procedure was used to create empirically derived $95 \%$ confidence intervals (Cls) for each group based on the observed sample data. Groups with $95 \% \mathrm{Cl}$ overlap are considered not significantly different from each other.

Conditioned suppression testing. Lever pressing during CS-on and CS-off phases were summed to create a total CS-on and total CSoff value. From this, a CS ratio was calculated as (total CS-on lever presses/total CS-on + total CS-off lever presses). A CS ratio of 0.50 indicates no change in lever pressing across CS-on and CS-off phases. CS ratios were analyzed in both behavioral and microinfusion studies using a priori, one-way, one-sample $t$ tests. This analysis determines whether the CS ratio for each group differs significantly from 0.5 , the value reflecting no conditioning suppression.

Electrophysiology. DA-induced changes in post-synaptic current peak amplitude were measured from baseline and are shown as percentages as follows: ((peak amplitude $\mathrm{DA}_{\mathrm{DA}}$ - peak amplitude $\left._{\text {baseline }}\right) /$ peak amplitude baseline $) \times 100$. Evoked $G_{A B A_{A^{-}}}$ IPSCs were binned ( $30 \mathrm{~s}$ ) and the amplitude data (baseline, DA application, and washout) were presented in an across-time analysis using an RM ANOVA. PPRs were calculated by dividing the second (S2) by the first (S1) peak amplitude. In cases in which sphericity was violated (Mauchley's $p<0.001$ ) the Greenhouse-Geisser correction was used (df rounded to the 
nearest whole number). Four recordings represented extreme outliers ( $>5$ SD) consistently for both amplitude and PPR, therefore these were removed from the analysis.

Correlation analysis. The relationship between sucrose intake and behavioral or electrophysiological measures was assessed using Pearson's $r$ correlations. First, the average amount of sucrose consumed (\% body weight) during the first hour of intake was summed over the past 4 days of sucrose consumption sessions for animals given intermittent access to sucrose ( $12 \mathrm{~h}$ sucrose). For animals tested in the behavioral experiment, this value was correlated with CS ratios; for those in the electrophysiology experiment, it was correlated with $G_{A B A_{A}}$-IPSC increases during DA application.

\section{RESULTS}

Sucrose consumption

Sucrose consumption was measured as solution $(\mathrm{ml})$ consumed per body weight ( $\mathrm{g}$ ) after the first hour of access, and at the end of 12 or $24 \mathrm{~h}$. For example, in Fig. $1 \mathrm{a}$, animals given $12 \mathrm{~h}$ access to sucrose consumed approximately $5 \%$ of their body weight in the first hour; for a $400 \mathrm{~g}$ rat, this would be $20 \mathrm{ml}$ in $1 \mathrm{~h}$. During the first hour of access, there was a significant interaction between session and group (Fig. 1a), $F_{26,1225}=1.98, p=0.003$, partial $\eta^{2}=$ 0.04 , indicating that animals with $12 \mathrm{~h}$ sucrose access escalated intake relative to, and consumed more solution than, $12 \mathrm{~h}$ saccharin or $24 \mathrm{~h}$ sucrose groups. There was also a significant session $\times$ group interaction for daily solution intake, $F_{15,708}=4.09$, $p<0.001$, partial $\eta^{2}=0.08$ (Fig. $1 \mathrm{~b}$ ): the two sucrose groups ( 12 and $24 \mathrm{~h}$ ) showed similar patterns of escalation and overall intake which were higher than daily solution consumption in the
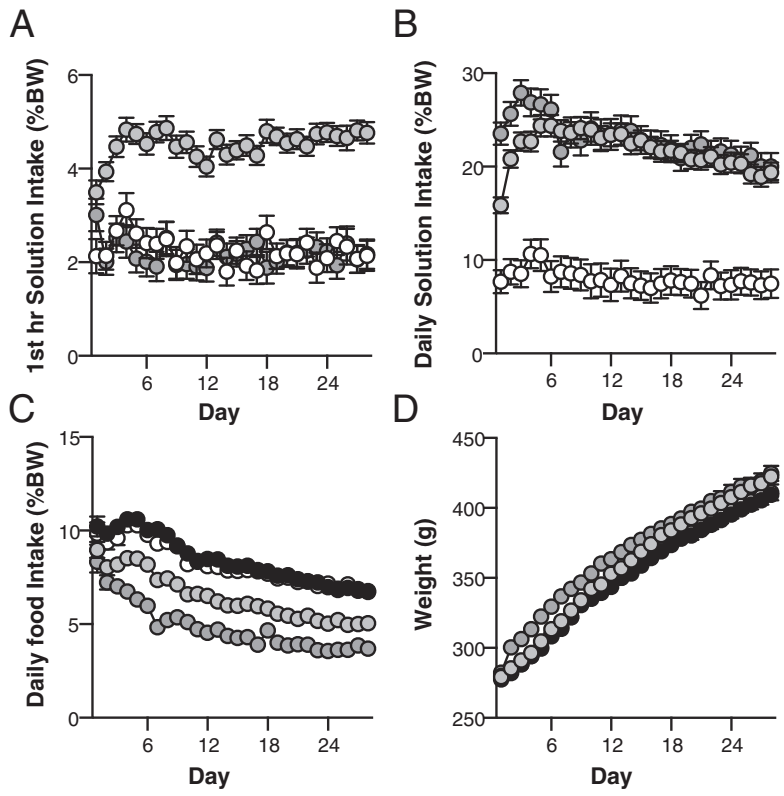

O $12 \mathrm{hr}$ Sucrose $12 \mathrm{hr}$ Food

$12 \mathrm{hr}$ Saccharin

$24 \mathrm{hr}$ Sucrose

Fig. 1 Solution and food intake across 28 days for rats given different periods of access to sucrose, saccharin, and food. Data are presented as group means and error bars represent standard error of the mean. a Mean solution intake $(\mathrm{ml})$ as a percentage of body weight $(\mathrm{g})$ during the first hour of each session. $\mathbf{b}$ Mean solution intake $(\mathrm{ml})$ as a percentage of body weight $(\mathrm{g})$ across each $24 \mathrm{~h}$ session. c Mean food intake (g) as a percent of body weight (g) across each $24 \mathrm{~h}$ session. d Mean body weight ( $\mathrm{g}$ ) across sessions. \% BW percentage body weight
A

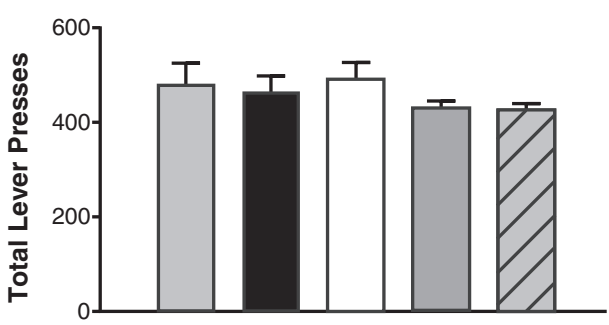

B

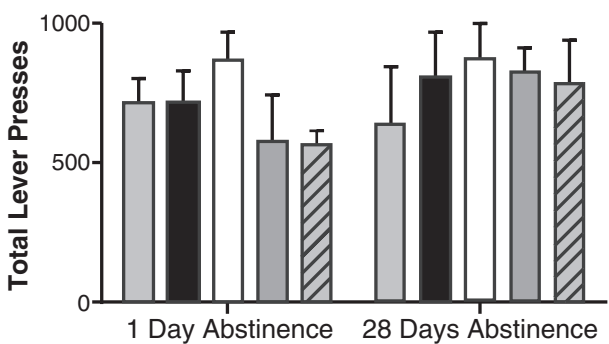

C

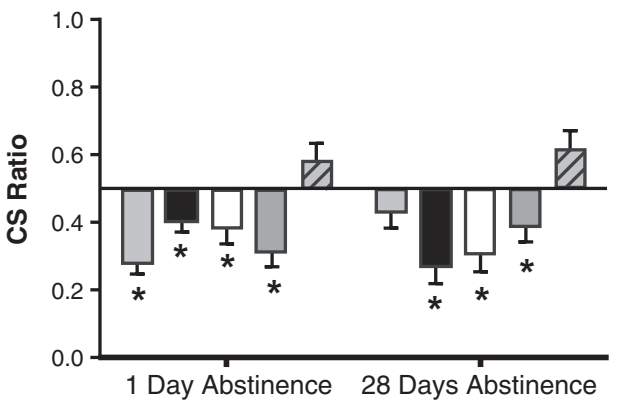

$\square 12 \mathrm{hr}$ Sucrose $\quad \square 12 \mathrm{hr}$ Food $\square 12 \mathrm{hr}$ Saccharin $\square 24 \mathrm{hr}$ Sucrose $\square \begin{gathered}12 \mathrm{hr} \text { Sucrose } \\ \text { CS Control }\end{gathered}$

Fig. 2 Lever pressing data during baseline, retraining, and conditioned suppression testing for groups given different periods of access to sucrose, saccharin, and food. Data are presented as group means and error bars represent $95 \% \mathrm{Cl}$ for each group (a, b), and standard error of the mean (C). a Lever pressing during the final session (baseline) of operant training under a fixed ratio (FR)5 schedule. b Lever pressing during an operant session (FR-5) following 1 or 28 days of sucrose abstinence. c Conditioned suppression responding following 1 or 28 days of sucrose abstinence. CS ratios were calculated as (total CS-on lever presses/ total CS-on + total CS-off lever presses). The vertical line at 0.5 represents consistent responding across CS-on and CS-off phases or a lack of conditioned suppression. *Values significantly lower than this value of $0.5, p<0.05$

$12 \mathrm{~h}$ saccharin group. Analysis of daily food consumption also revealed a significant session $\times$ group interaction, $F_{21,889}=198.25$, $p<0.001$, partial $\eta^{2}=0.06$ (Fig. 1c), in that food intake (relative to body weight) decreased over sessions, with the two sucrose groups consuming less food than either the $12 \mathrm{~h}$ saccharin or food only groups. The significant session $\times$ group interaction for daily weight, $F_{6,232}=2.53, p=0.025$, partial $\eta^{2}=0.06$, was due to the fact that weight increased across sessions for all groups, but the $24 \mathrm{~h}$ sucrose group was heavier than the other groups in the first half of the sessions (Fig. 1d). By the end of the sucrose consumption sessions (28 days), body weights of all groups were similar.

\section{Experiment 1: Behavior}

There were no group differences in the acquisition of operant responding across training or in the total number of lever pressing responses, $p s>0.05$, indicated by overlapping $95 \% \mathrm{Cls}$ (Fig. 2a). Forced abstinence did not alter these responses in that rates of 
A
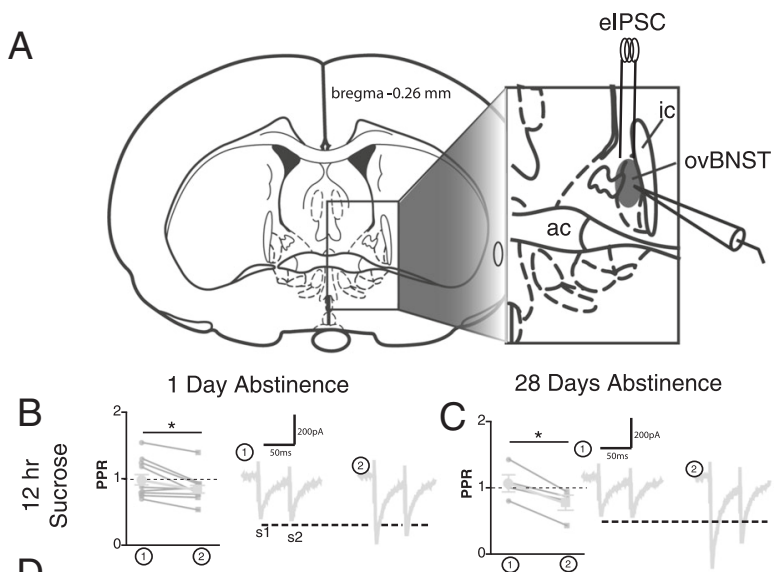

1 Day Abstinence

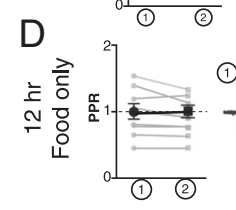

F

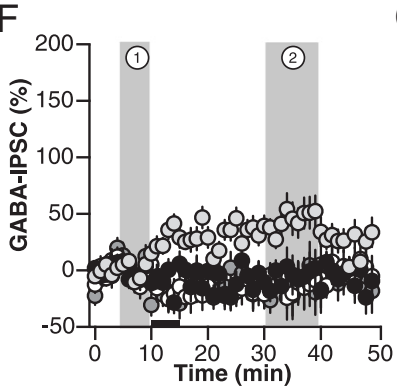

$012 \mathrm{hr}$ Sucrose $\bullet 12 \mathrm{hr}$ Food Only
28 Days Abstinence

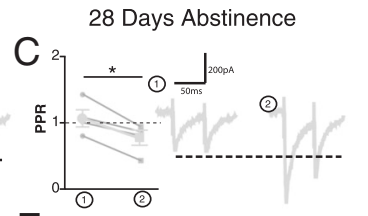

$\frac{1}{50 m s}^{200 \mathrm{pA}}$

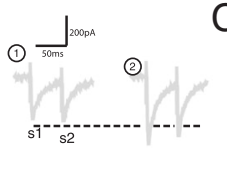

1

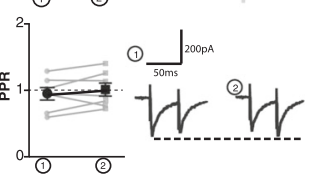

G

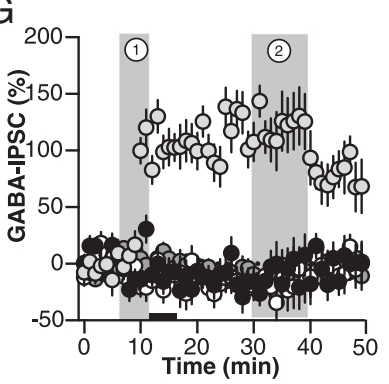

$012 \mathrm{hr}$ Saccharin $024 \mathrm{hr}$ Sucrose

Fig. 3 Effect of dopamine (DA) application (5 min) on inhibitory synaptic transmission of ovBNST neurons of rats given different periods of access to sucrose, saccharin, and food. a Schematic illustration of the experimental setting for ovBNST (shaded area in the inset) whole-cell voltage-clamp recordings of $G A B A_{A}$-inhibitory post-synaptic currents (IPSC). Summary of effects of DA on PPR of GABAA-IPSC in the following groups: $12 \mathrm{~h}$ sucrose (b) 1-day abstinence and c 28-day abstinence; $12 \mathrm{~h}$ food (d) 1-day abstinence and e 28-day abstinence. Each gray circle represents an individual neuron and black circles/squares are means \pm SEM of PPRs at times 0 and $30 \mathrm{~min}$. Representative traces of electrically evoked GABAA-IPSC before (left traces) or after (right traces) bath application DA (1 $\mu \mathrm{M})$ are shown next to the summary of effects. S1 and S2 represent the maximum $\mathrm{GABA}_{\mathrm{A}}$-IPSC amplitude in response to two electrical stimulations given at $40 \mathrm{~Hz}$ to measure changes in paired-pulse ratios (data reported in Results section). Effect of bath application of DA ( $1 \mu \mathrm{M}$, black horizontal bar) on the change in amplitude of electrically evoked GABAA-IPSC amplitude as a function of time following (f) 1-day abstinence or g 28-day abstinence. Data are presented as mean of $30 \mathrm{~s}$ bins (5 events/bin) and error bars represent standard error of the mean

responding were similar across groups during post-abstinence testing, again based on $95 \%$ Cls (Fig. 2b).

As shown in Fig. 2c, only the CS Unpaired group did not exhibit conditioned suppression following 1 day of sucrose abstinence. Following 28 days of abstinence, however, animals given intermittent access to sucrose also failed to inhibit responding when a cue predicting food shock (CS-on) was presented. This effect was verified by one-way a priori $t$ tests indicating that the $12 \mathrm{~h}$ sucrose plus 28-day abstinence group showed resistance to conditioned suppression, $t_{87}=-1.14, p=0.128$, and lever pressed at a similar rate as animals that did not learn the tone-shock contingency in the CS task, $t s_{87} \geq 1.45, p s \leq 0.076$. All other groups

showed significant suppression of responding during CS-on phases, $t \mathrm{~s}_{87} \leq 1.98, \mathrm{ps} \leq 0.025$.

Experiment 2: Electrophysiology

To determine if DA differentially affected synaptic activity in the ovBNST of animals based on sucrose access schedules and/or time spent abstinent, the effect of $1 \mu \mathrm{M}$ DA on the amplitude of electrically evoked $G_{A B A_{A}-I P S C}$ was measured. This analysis revealed a significant three-way interaction of time, group, and abstinence length on evoked IPSCs (Fig. 3a, b), $F_{32,636}=1.48, p=$ 0.046 , partial $\eta^{2}=0.07$. Specifically, bath application of $D A$ reversibly increased (maximum $+54 \%$ ) the amplitude of $G_{A B A}$ IPSCs in the ovBNST of the $12 \mathrm{~h}$ sucrose group following 1 day of abstinence (Fig. 3b, c), but produced a large and long-lasting (maximum $+138 \%$ ) increase in the $12 \mathrm{~h}$ sucrose group following 28 days of abstinence (Fig. 3b, c); there was a modest reduction in the amplitude of electrically evoked $G_{A B A}-I P S C$ in all other groups (Fig. 3b, c). In other words, the effects of DA on GABA ${ }_{A}$ IPSC amplitude differed across groups, only producing a significant increase in this measure for animals given $12 \mathrm{~h}$ access to sucrose, and this increase was significantly enhanced in groups that experienced 28 days of abstinence.

We conducted PPR analyses to determine whether DA acted pre-synaptically or post-synaptically to modulate ovBNST GABA transmission. DA significantly reduced PPR in ovBNST neurons of the intermittent sucrose access groups regardless of abstinence length (time $\times$ group interaction, $F_{5,93}=5.21, p<0.001$, partial $\eta^{2}=0.21$ ) Likewise, DA increased PPR in both the intermittent food and saccharin groups, although no change was observed for animals with ad libitum sucrose access ( $24 \mathrm{~h}$ sucrose). In sum, DA pre-synaptically modulated GABA transmission in the ovBNST which is consistent with our previous observations [29-31]. Consistent with the effect of cocaine we previously observed, the polarity but not the location of DA modulation of ovBNST GABA transmission changed with intermittent sucrose access.

Behavioral and electrophysiological correlates

Sucrose consumption (\% of body weight) during the first hour of the last four consumption sessions was positively related to conditioned suppression (i.e., CS ratios) for animals in the intermittent sucrose group, Pearson's $r(n=21)=0.433, p=0.05$. That is, greater sucrose consumption was associated with higher CS ratios or reduced conditioned suppression. No other group exhibited a significant relationship between solution intake and CS ratio, $12 \mathrm{~h}$ saccharin, $r(n=16)=-0.08, p=0.745 ; 24 \mathrm{~h}$ sucrose, $r(n=16)=0.398, p=0.127 ; 12 \mathrm{~h}$ sucrose CS control, $r(n=16)=$ $-0.04, p=0.884$, (Fig. 4a). When data from the $12 \mathrm{~h}$ sucrose group was divided into abstinence conditions, we found a significant correlation following 1-day, $r(\mathrm{n}=13)=0.728, p=0.005$, but not 28-day, $r(\mathrm{n}=8)=-0.211, p=0.616$, of abstinence. In other words, the magnitude of conditioned suppression was sensitive to the amount of sucrose consumed after 1 day of abstinence but became largely inflexible after 28 days.

Similarly, the amount of sucrose consumed during the first hour of access was positively related to $\mathrm{GABA}_{A}$ IPSCs for animals in the intermittent sucrose group only, Pearson's $r(n=12)=0.724$, $p=0.004$. Neither the intermittent saccharin, Pearson's $r(n=14)$ $=0.033, p=0.456$, nor the ad libitum sucrose, Pearson's $r(n=21)$ $=-0.045, p=0.422$, groups showed a significant relationship (Fig. 4b). Combined, these results indicate that the severity of sucrose intake in rats given intermittent access to sucrose was positively related to the severity of both compulsive responding for sucrose-related cues and synaptic $G_{A B A_{A}}$ activity in the ovBNST.

Experiment 3: Microinfusion

Cannulae placements for animals receiving SCH-23990 infusions into the ovBNST are shown in Fig. 5a. All saline infusions fell within 

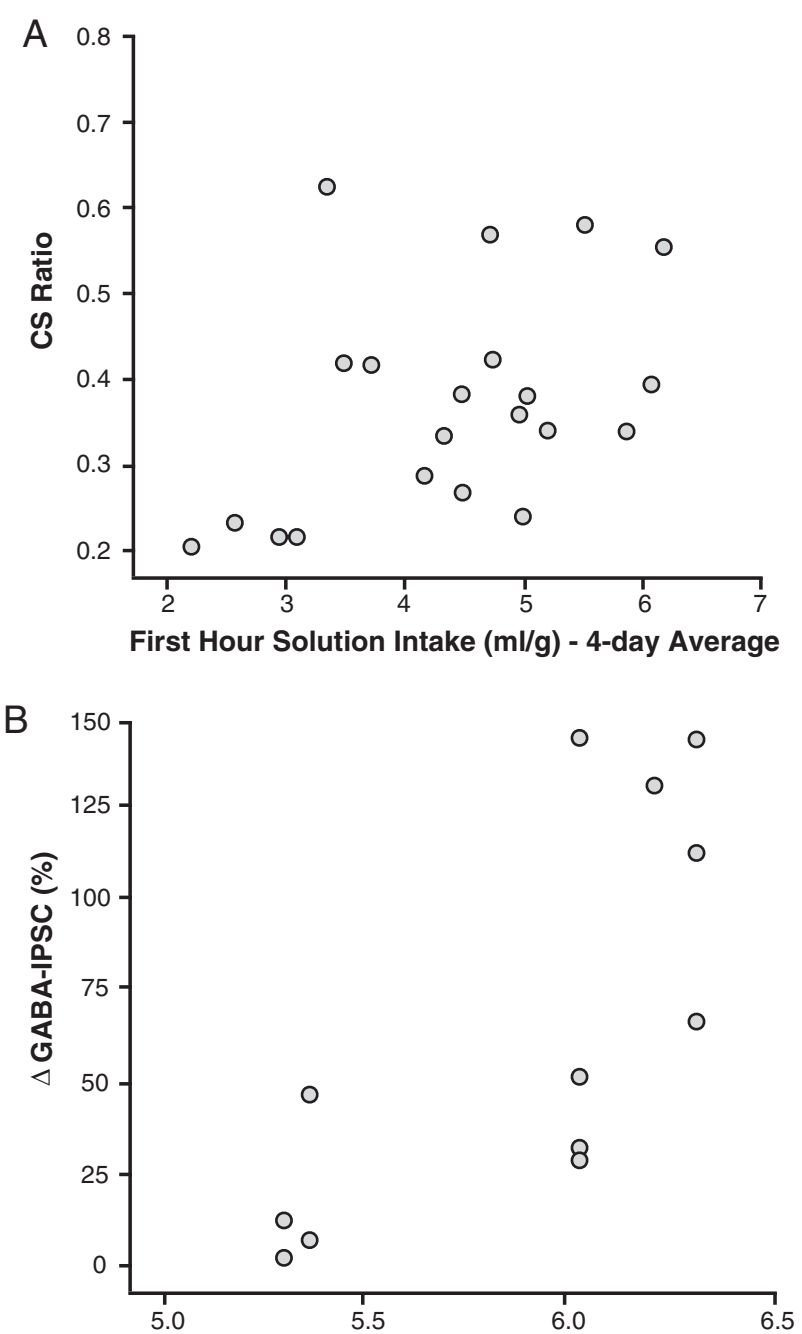

First Hour Solution Intake $(\mathrm{ml} / \mathrm{g})$ - 4-day Average

Fig. 4 Relationship between sucrose consumption and behavioral and electrophysiological measures for animals given intermittent $(12 \mathrm{~h})$ access to sucrose over 28 days. The relationship between amount of sucrose consumed during the first hour of access over the last four consumption sessions and a suppression of responding in the conditioned suppression task or $\mathbf{b}$ magnitude of change in $\mathrm{GABA}_{\mathrm{A}}$ IPSCS

the ovBNST boundaries as shown in Fig. 5a. During testing (see Fig. 5b), all groups that received microinfusions of saline into the ovBNST displayed CS, $t s_{56} \geq 2.79, p s \leq 0.004$, with the exception of the $12 \mathrm{~h}$ sucrose group, $t_{56}=0.12, p=0.45$. Conversely, all groups exhibited conditioned suppression following microinfusions of $0.8 \mu \mathrm{g} / \mu \mathrm{l} \mathrm{SCH}-23390, t \mathrm{~s}_{56} \geq 2.00, p \mathrm{~s} \leq 0.025$, although the effect was marginal in the $12 \mathrm{~h}$ food group, $t_{56}=1.63, p=0.055$. Infusions of SCH-23990 that reversed the blockade of conditioned suppression (i.e., $12 \mathrm{~h}$ sucrose group) were not confined to a specifically anatomical region within the ovBNST (see Fig. 5a).

To ensure that $\mathrm{SCH}-23390$ was not interfering with locomotor responses, latency to lever press during testing was analyzed using a two-way ANOVA with drug and intake group as between subject's factors (see Fig. 5c). Data were log transformed to maintain homogeneity of variance. This analysis revealed no effect of group, $F_{(3,52)}=1.52, p=0.221$, or drug, $F_{(1,52)}=3.97, p=0.52$, and no interaction between the two, $F_{(3,52)}=0.55, p=0.651$. Combined, these data indicate that blockade of conditioned suppression in animals given intermittent access to sucrose was
A

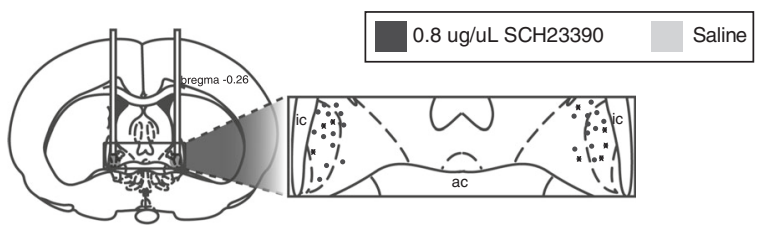

B

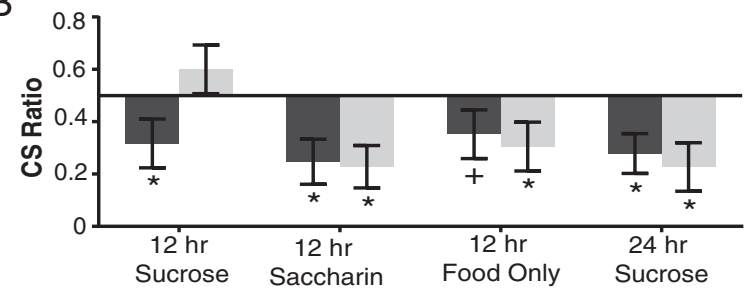

C

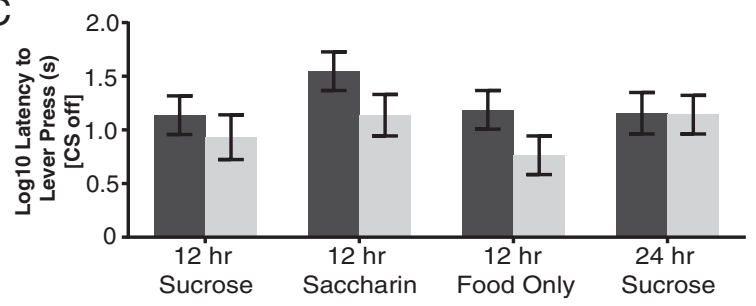

Fig. 5 Effects of intra-ovBNST DA D1 receptor blockade on conditioned suppression responding. a Drawing showing correct intracranial cannulae placements for rats receiving $0.8 \mu \mathrm{g} / \mu \mathrm{l} \mathrm{SCH}-$ 23990 infusions. For clarity, each bilateral infusion is represented unilaterally (total $n=32$ ) and infusions in the $12 \mathrm{~h}$ sucrose consumption group are identified with $\mathrm{X}(n=8)$. b Conditioned suppression (CS) ratios and $c$ latency to lever press during CS testing for rats in different access groups following ovBNST infusions of $\mathrm{SCH}-23990$ or saline. CS ratios were calculated as (total CS-on lever presses/total CS-on + total CS-off lever presses). The vertical line at 0.5 represents consistent responding across CS-on and CS-off phases or a lack of conditioned suppression. *Values significantly lower than this value of $0.5, p<0.05$

reversed by microinfusions of $\mathrm{SCH}-23390$ into the ovBNST, without affecting gross motor responses.

\section{DISCUSSION}

Consistent with previous reports [16, 33], animals given intermittent access to sucrose displayed marked bingeing behavior, evidenced by a robust increase in solution consumption during the first hour of access. This behavior emerged within 3 days and was maintained across 28 days of testing. Notably, animals with limited access to sucrose consumed the same amount per day as those with ad libitum access, although close to one-fourth of this daily intake occurred within the first hour of access. Rats given intermittent access to saccharin did not develop binge-like intake, although this behavior has been observed in mice using a lower concentration of saccharin [34]. Food intake decreased over sessions in all groups, reflecting reduced caloric requirements of rats that have reached full maturity. Although there were no group differences in body weight across testing, animals given access to sucrose ate less food on a daily basis, presumably to compensate for the calories they consumed from sucrose [16].

The most compelling behavioral effect in our study was the resistance to conditioned suppression in animals given intermittent access to sucrose, matching behaviors that develop following self-administration of cocaine $[14,35]$, or high-fat diets $[15,36]$. Compulsive responding in our experiment was fully detectable after 28 days of forced abstinence, but less reliable immediately following sucrose access. This suggests that our 
sucrose bingeing protocol was less robust than those producing compulsive responding for cocaine or high fat, as these latter effects are observed within days of the last intake session. However, our findings are consistent with cocaine-induced effects in that compulsive responding was enhanced following abstinence [35], supporting the idea that biological changes underlying altered responding for reward-paired cues are not drug-specific [37].

In parallel to our behavioral findings, only animals with intermittent access to sucrose displayed a significant increase in $G_{A B A}-I P S C s$ in ovBNST neurons following DA application. This increase was markedly larger for animals that experienced 28 days of forced abstinence and, as with compulsive responding, sucrose consumption was positively correlated with increases in the magnitude of $G_{A B A}-$ IPSCs. These results are strikingly similar to those in animals that chronically self-administer cocaine $[28,30,31]$, suggesting that there are common processes modulating the control of substance intake in the ovBNST. Based on our previous research, we propose that these involve a concurrent increase in post-synaptic DRD1 levels and a decrease in pre-synaptic DA D2 receptor (DRD2) function [29-31]. More specifically, in baseline states, DA binds to pre-synaptic DRD2 receptors which reduces GABA release and decreases $G_{A B A}-I P S C s$ [29]. Following sucrose bingeing, pre-synaptic DRD2 receptors lose functionality, and DRD1 receptors accumulate post-synaptically $[29,31]$. Activation of these post-synaptic DRD1 receptors then produces a cascading increase in GABA IPSCs (DRD1-LTP $_{G A B A}$ ) via a feedback mechanism involving post-synaptic release of neurotensin [30]. A similar process has been reported in other brain regions: binge intake of high-fat food produces compulsive responding that is matched by a reduction in DRD2 density in the striatum [15], and the effect may be mediated by GABA-DRD2 receptor interactions in the medial prefrontal cortex [38].

The mechanism by which BNST neurons contribute to compulsive responding for sucrose likely involves connections to the lateral hypothalamus (LH), a region long implicated in feeding [39]. Food intake activates BNST neurons [40] and stimulation of a BNST-LH circuit induces voracious feeding that is preferentially directed to highly palatable food [26]. Based on this evidence, Jennings et al. [26] propose that inhibitory connections from the ovBNST suppress activity of LH neurons to control food intake. Disruptions in this process, including altered DAergic regulation of ovBNST GABA transmission, would lead to uncontrolled food intake. Notably, we did not observe any change in ovBNST responses to DA in non-bingeing animals exposed to sucrose (i.e., $24 \mathrm{~h}$ group), matching evidence that BNST modulation of food intake is not due to non-selective effects on feeding [27]. Importantly, our finding that sucrose bingeing animals displayed conditioned suppression following blockade of DRD1 receptors in the ovBNST is evidence for a causal link between the emergence of compulsive responding and alterations in DAergic mechanisms in the BNST. It is unlikely that the effects of DRD1 receptor blockade were nonspecific because latencies to lever press were not altered by this manipulation and our previous work confirms that SCH-23390 infusions into the ovBNST do not alter lever pressing for sucrose [30]. Moreover, systemic administration of the drug do not affect operant responding under a variety of reinforcement schedules [41].

Given that both behavioral and electrophysiological effects of sucrose bingeing were enhanced following abstinence, our original goal was to conduct a microinfusion study using the same delay ( 28 days). Unfortunately, the negative health impact of combining excessive sucrose intake with intracranial surgery eliminated this option. The high mortality rate in this experiment may not be surprising as excessive sucrose intake leads to compromised immune system function [42] and the BNST could play an important role in this process $[25,43]$. Because of this change in experimental design, we observed an apparent discrepancy in our data: blockade of conditioned suppression following 1 day of abstinence in the microinfusion saline group, but not in the original behavioral experiment (first bar of Fig. $2 \mathrm{c}$ versus second bar of Fig. 5b). This may simply reflect variability, particularly as our preliminary experiments consistently revealed a more pronounced effect of sucrose bingeing on conditioned suppression with longer periods of abstinence. The pattern was confirmed in our electrophysiological studies, which showed similar alterations in DA mechanisms after 1 and 28 days, but stronger effects in the latter. Alternatively, cannulae implantation itself may have altered reward and/or immune system processes in the BNST, thereby altering conditioned suppression responses in the saline group.

The BNST role in controlling food intake likely reflects a broader function of this brain region in emotional regulation related to motivated behaviors [44]. Anatomically, the BNST is positioned to integrate autonomic, neuroendocrine, and behavioral responses and may function to re-establish homeostasis in the face of physiological or environmental challenge [45]. This fits with evidence that the BNST has a primary role in stress-related and anxiety-related behaviors [46-48]. Disruption of these processes would be manifested as an inability to regulate responses to emotionally laden stimuli, both rewarding and aversive. If BNST function is compromised following excessive intake of highly palatable food (as in our model), it would help to explain why deficits in emotional dysregulation are primary characteristics of BED [49] and one of the most common explanations for binge eating in humans [50]. We should note, however, that our study included only male rats so the findings may not transfer to females, particularly as the BNST is sexually dimorphic at both anatomical and neurochemical levels [51]. This is an important direction for further work given the increased prevalence of eating disorders in females [2].

Finally, our data may have broader implications for understanding compulsion that occurs in other psychiatric disorders, such as drug addiction. Repeated consumption of high-sugar or high-fat foods, like chronic self-administration of stimulant drugs, may shift the control of behavior from an action-outcome to a habit-driven system [52]. If so, compulsive behavior would emerge as individuals progress from controlled self-regulated intake of sugar or drugs to uncontrolled and disinhibited use. The fact that we observed changes in BNST function following sucrose bingeing that parallel those reported in rats with a history of cocaine intake $[29,31]$ supports the idea of a common dysregulatory process in maladaptive eating and drug use. Moreover, recent conceptualizations of BNST function focus on addiction-related disorders $[44,53]$. At the same time, eating and substance-related disorders are distinct clinical categories, at least in the most recent version of DMS-5, and a number of researchers question the overlap between mechanisms that underlie food and drug addiction $[10,11,54-56]$. Thus, our findings are most pertinent to BED and associated problems, such as obesity, although the work may ultimately inform public health initiatives related to other conditions, including substance abuse.

\section{ACKNOWLEDGEMENTS}

We thank Nicole Avena for comments on the manuscript and Lisa Wilberforce and Roland Dupras for technical assistance. Queen's University is situated on traditional Anishinaabe and Haudenosaunee Territory. This work was supported by a Discovery Grant from the Natural Sciences and Engineering Research Council of Canada (NSERC) to M.C.O. (203707), an NSERC graduate fellowship to A.C.M., a Canadian Institutes of Health Research (CIHR) Operating Grant to É.C.D. (MOP-25953), and a CIHR Vanier Graduate Fellowship to C.P.N.

\section{ADDITIONAL INFORMATION}

Competing interests: The authors declare no competing interests. 
Publisher's note: Springer Nature remains neutral with regard to jurisdictional claims in published maps and institutional affiliations.

\section{REFERENCES}

1. Hudson Jl, Hiripi E, Pope HG Jr, Kessler RC. The prevalence and correlates of eating disorders in the national cormorbidity survbey repcliation. Biol Psychiatry. 2007;61:348-58

2. Kessler RC, Berglund PA, Chiu WT, Deitz AC, Hudson Jl, Shahly V, et al. The prevalence and correlates of binge eating disorder in the WHO World Mental Health Surveys. Biol Psychiatry. 2013;73:904-14.

3. American Psychiatric Association. Diagnostic and statistical manual of psychiatric disorders. 5th ed. Arlington, VA: American Psychiatric Publishing; 2013. (DSM-5)

4. Stojek MMK, MacKillop J. Relative reinforcing value of food and delayed reward discounting in obesity and disordered eating: a systematic review. Clin Psychol Rev. 2017;55:1-11.

5. Rieger E, Wilfley DE, Stein RI, Marino V, Crow SJ. A comparison of quality of life in obese individuals with and without binge eating disorder. Int J Eat Disord. 2005;37:234-40.

6. Davis C, Curtis C, Levitan RD, Carter JC, Kaplan AS, Kennedy JL. Evidence that 'food addiction' is a valid phenotype of obesity. Appetite. 2011;57:711-7.

7. Gearhardt AN, White MA, Masheb RM, Morgan PT, Crosby RD, Grilo CM. An examination of the food addiction construct in obese patients with binge eating disorder. Int J Eat Disord. 2012;45:657-63.

8. Burmeister JM, Hinman N, Koball A, Hoffmann DA, Carels RA. Food addiction in adults seeking weight loss treatment. Implications for psychosocial health and weight loss. Appetite. 2013;60:103-10.

9. Clark SM, Saules KK. Validation of the Yale food addiction scale among a weightloss surgery population. Eat Behav. 2013;14:216-9.

10. Treasure J, Leslie $M$, Chami R, Fernández-Aranda F. Are trans diagsnotic models of eating disorders fit for purpose? A consideration of the evidence for food addiction. Eur Eat Disord Rev. 2018;26:83-91.

11. Westwater ML, Fletcher PC, Ziauddeen H. Sugar addiction: the state of the science. Eur J Nutr. 2016;55:55-69.

12. Hutson PH, Balodis IM, Potenza MN. Binge-eating disorder: clinical and therapeutic advances. Pharmacol Ther. 2018;182:15-27.

13. Corwin RL, Avena NM, Boggiano MM. Feeding and reward: perspectives from three rat models of binge eating. Physiol Behav. 2011;104:87-97.

14. Vanderschuren LJMJ, Everitt BJ. Drug seeking becomes compulsive after prolonged cocaine self-administration. Science. 2004;305:1017-9.

15. Johnson PM, Kenny PJ. Dopamine D2 receptors in addiction-like reward dysfunction and compulsive eating in obese rats. Nat Neurosci. 2010;13:635-41.

16. Avena NM, Rada P, Hoebel BG. Evidence for sugar addiction: behavioral and neurochemical effects of intermittent, excessive sugar intake. Neurosci Biobehav Rev. 2008;32:20-39.

17. Grimm JW, Fyall AM, Osincup DP. Incubation of sucrose craving: effects of reduced training and sucrose pre-loading. Physiol Behav. 2005;84:73-79.

18. Avena NM, Long KA, Hoebel BG. Sugar-dependent rats show enhanced responding for sugar after abstinence: evidence of a sugar deprivation effect. Physiol Behav. 2005;84:359-62.

19. Aoyama K, Barnes J, Grimm JW. Incubation of saccharin craving and withinsession changes in responding for a cue previously associated with saccharin. Appetite. 2014;72:114-22.

20. Grimm JW, Hope BT, Wise RA, Shaham Y. Incubation of cocaine craving after withdrawal. Nature. 2001;412:141-2.

21. Shalev U, Morales M, Hope B, Yap J, Shaham Y. Time-dependent changes in extinction behavior and stress-induced reinstatement of drug seeking following withdrawal from heroin in rats. Psychopharmacology (Berl). 2001;156:98-107.

22. Pickens $\mathrm{Cl}$, Airavaara M, Theberge F, Fanous S, Hope BT, Shaham Y. Neurobiology of the incubation of drug craving. Trends Neurosci. 2011;34:411-20.

23. Ciccocioppo R, Cippitalli A, Economidou D, Fedeli A, Massi M. Nociception/ orphanin FQ acts as a functional antagonist of corticotropin-releasing factor to inhibit is anorectic effect. Physiol Behav. 2004;82:63-68.

24. Ciccocioppo R, Fedeli A, Economidou D, Policani R, Weiss F, Massi M. The bed nucleus is a neuroanatomical substrate for the anorectic effect of corticotropinreleasing factor and for its reversal by nociceptin/orphanin FQ. J Neurosci. 2003;23:9445-51.

25. Francesconi W, Sánchez-Alavez M, Berton F, Alboni S, Benatti C, Mori S, et al. The proinflammatory cytokine interleukin 18 regulates feeding by acting on the bed nucleus of the stria terminalis. J Neurosci. 2016:36:5170-80.

26. Jennings JH, Rizzi G, Stamatakis M, Ung UL, Stuber GD. The inhibitory circuit architecture of the lateral hypothalamus orchestrates feeding. Science. 2013;341:1517-21.

27. Micioni Di Bonaventura MV, Ciccioppo R, Romano A, Bossert JM, Rice KC, Ubaldi $M$, et al. Role of bed nucleus of the stria terminalis corticotrophin-releasing factor receptors in frustration stress-induced binge-like palatable food consumption in female rats with a history of food restriction. J Neurosci. 2014;34:11316-24.

28. Krawczyk M, Debacker J, Mason X, Jones AA, Dumont ÉC. Dopamine decreases NMDA currents in the oval bed nucleus of the stria terminalis of cocaine self-administering rats. Prog Neuropsychopharmacol Biol Psychiatry. 2014:51C:83-88.

29. Krawczyk M, Georges F, Sharma R, Mason X, Berthet A, Bézard E, et al. Doubledissociation of the catecholaminergic modulation of synaptic transmission in the oval bed nucleus of the stria terminalis. J Neurophysiol. 2011;105:145-53.

30. Krawczyk M, Mason X, DeBacker J, Sharma R, Normandeau CP, Hawken ER, et al. D1 dopamine receptor-mediated LTP at GABA synapses encodes motivation to self-administer cocaine in rats. J Neurosci. 2013;33:11960-119671.

31. Krawczyk M, Sharma R, Mason X, DeBacker J, Jones AA, Dumont ÉC. A switch in the neuromodulatory effects of dopamine in the oval bed nucleus of the stria terminalis associated with cocaine self-administration in rats. J Neurosci. 2011;31:8928-35.

32. Paxinos G, Watson C. The rat brain in stereotaxic coordinates. 7th ed. Cambridge, MA: Academic Press; 2013.

33. Hoebel BG, Avena NM, Bocarsly ME, Rada P. Natural addiction: a behavioral and circuit model based on sugar addiction in rats. J Addict Med. 2009;3:33-41.

34. Yasoshima $Y$, Shimura T. A mouse model for binge-like sucrose overconsumption: contribution of enhanced motiviation for sweetner consumption. Physiol Behav. 2015;138:154-64.

35. Gancarz-Kausch QM, Adank DN, Dietz DM. Prolonged withdrawal following cocaine self-administration increaswes resistance to punishment in a cocaine binge. Sci Rep. 2014;4:6876.

36. Heyne A, Kiesselbach C, Sahún I, McDonald J, Gaiffi M, Dierssen M, et al. An animal model of compulsive food-taking behaviour. Addict Biol. 2009;14:373-83.

37. Lu L, Grimm JW, Hope BT, Shaham Y. Incubation of cocaine craving after withdrawal: a review of preclinical data. Neuropharmacology. 2004;47 Suppl 1:214-26.

38. Corwin RL, Wojnicki FHE, Zimmer DJ, Babbs RK, McGrath LE, Olivos DR, et al. Binge-type eating disrupts dopaminergic and GABAergic signaling in the prefrontal cortex and ventral tegmental area. Obesity. 2016;24:2118-25.

39. Hoebel BG, Teitelbaum P. Hypothalamic control of feeding and self-stimulation. Science. 1962;135:375-7.

40. Ángeles-Castellanos M, Mendoza J, Escobar C. Restricted feeding schedules phase shift daily rhythms of c-Fos and protein Per1 immunoreactivity in corticolimbic regions in rats. Neuroscience. 2007;144:344-55.

41. Feltmann K, Giuliano C, Everitt BJ, Steensland P, Alsiö J. The effects of the monoamine stabilizer (-)-OSU6162 on binge-like eating and cue-controlled food-seeking behavior in rats. Neuropsychopharmacology. 2018;43:617-26.

42. Hotamisligil GS. Foundations of immunometabolism and implications for metabolic health and disease. Immunity. 2017:47:406-20.

43. Bienkowski MS, Rinaman L. Immune challenge activates neural inputs to the ventrolateral bed nucleus of the stria terminalis. Physiol Behav. 2011;104:257-65.

44. Stamatakis AM, Sparta DR, Jennings JH, McElligott ZA, Decot $H$, Stuber GD. Amygdala and bed nucleus of the stria terminalis circuitry: implications for addiction-related behaviors. Neuropharmacology. 2014;76 Pt B:320-8.

45. Crestani CC, Alves FHF, Gomes FV, Resstel LBM, Correa MFA, Herman JP. Mechanisms in the bed nucleus of the stria terminalis involved in control of autonomic and neuroendocrine functions: a review. Curr Neuropharmacol. 2013;11:141-59.

46. Kim SY, Adhikari A, Lee SY, Marchel JH, Kim Ck, Mallory CS, et al. Diverging neural pathways assemble a behavioural state from separable features in anxiety. Nature. 2013;496:219-23.

47. Normandeau DP, Ventura-Silva AP, Hawken ER, Angelis S, Sjaarda C, Liu X, et al. A key role for neurotensin in chronic-stress-induced anxiety-like behavior in rats. Neuropsychopharmacology. 2017;134:353-8.

48. Silberman $Y$, Winder DG. Emerging role for corticotropin releasing factor signaling in the bed nucleus of the stria terminalis at the intersection of stress and reward. Front Psychiatry. 2013;4:1-12.

49. Heatherton TF, Wager DD. Cognitive neuroscience of self-regulation failure. Trends Cogn Sci. 2011;15:132-9.

50. Leehr EJ, Krohmer K, Schag K, Dresler T, Zipfel S, Giel KE. Emotion regulation model in beinge eating disorder and obesity-a systemaic review. Neurosci Behav Rev. 2015:49:125-34.

51. King SB, Lezak KR, O'Reilly M, Toufexis DJ, Falls WA, Braas K, et al. The effects of prior stress on anxiety-like responding to intra-BNST pituitary adenylate cylase activating polypeptide in male and female rates. Neuropsychopharmacol. 2017;42:1679-87.

52. Smith DG, Robbins TW. The neurobiological underpinnings of obesity and binge eating: a rationale for adopting the food addiction model. Biol Psychiatry. 2013;73:804-10. 
Dopamine in the oval bed nucleus of the stria terminalis contributes to... AC Maracle et al.

53. Avery SN, Clauss JA, Winder DG, Woodward N, Heckers S, Blackford JU. BNST neurocircuitry in humans. Neuroimage. 2014;1:311-23.

54. Corwin RL, Hayes J. Are sugars addictive? Perspectives for practitioners. In: Rippe $\mathrm{J}$, editors. Fructose, high fructose corn syrup, sucrose and health. New York: Humana Press; 2014. p. 199-215.
55. DeLeone RJ, Taylor JR, Picciotto MR. The drive to eat: comparisons and distinctions between mechanisms of food reward and drug addiction. Nat Neurosci. 2012;15:1330-5.

56. Ziauddeen H, Farooqi IS, Fletcher PC. Obesity and the brain: how convincing is the addiction model? Nat Rev. 2012;13:279-86. 